\title{
Development of 4-Dimensional Human Model System for the Patient after Total Hip Arthroplasty
}

\author{
Yoshito Otake $^{1}$, Keisuke Hagio ${ }^{2}$, Naoki Suzuki ${ }^{1}$, Asaki Hattori ${ }^{1}$, \\ Nobuhiko Sugano ${ }^{3}$, Kazuo Yonenobu ${ }^{4}$, and Takahiro Ochi ${ }^{2}$ \\ ${ }^{1}$ Institute for High Dimensional Medical Imaging, Jikei University School of Medicine, \\ 4-11-1, Izumi Honcho, Komae-shi, Tokyo, Japan \\ \{otake, nsuzuki, hat\}@jikei.ac.jp \\ ${ }^{2}$ Department of Computer Integrated Orthopaedics, \\ Osaka University Graduate School of Medicine, \\ 2-2 Yamadaoka, Suita 565-0871, Osaka, Japan \\ k-hagio@caos.med.osaka-u.ac.jp, ochi@ort.med.osaka-u.ac.jp \\ ${ }^{3}$ Department of Orthopaedic Surgery, Osaka University Graduate School of Medicine, \\ 2-2 Yamadaoka, Suita 565-0871, Osaka, Japan \\ sugano@ort.med.osaka-u.ac.jp \\ ${ }^{4}$ Department of Orthopaedic Surgery, Osaka Minami National Hospital, \\ 2-1 Kidohigasi-machi, Kawachinagano 586-0008, Osaka, Japan \\ yonenobu-k@umin.ac.jp
}

\begin{abstract}
In total hip arthroplasty(THA), complications such as dislocation, loosening, or wearing of the sliding surface are serious clinical problem, and the daily motion of patients has been limited to some extent. However, it is hard to recognize the situation of the components during movement and to predict complications. We have developed the 4-dimensional human model that can visualize the motion of the patient's skeleton and estimate the risk of complications using computer simulation. At first we constructed a 3-dimensional skeletal model of the patient's lower limb from CT data. Then we acquired motion capture data from an infrared position sensor (VICON512, VICON Motion Systems, UK), and drove the patient's skeletal model corresponding to the captured data. Thus we were able to predict the prognosis after the installation of the artificial hip joint, and we have examined the accuracy of the measurements in this system following an experiment using an open MRI.
\end{abstract}

\section{Background}

Generally, the daily motion of patients who underwent total hip arthroplasty (THA) has been limited to some extent in order to prevent complications such as dislocation, loosening, or wearing[1][2][3][4]. However the causes of these complications are related to the individual patient's information regarding the position and alignment of the components, skeletal structure, and characteristics of the daily motion. Therefore to give a precise guidance of postoperative daily motion for each patient is difficult for a clinician. The effects of the component alignment on the range of hip motion were reported previously[5][6], but the relative position of the components and skeletal structures during movement has not been reported. 


\section{Purpose}

The purpose of this study is (1)to develop a 4-dimensional motion analysis system that can visualize the patient's skeletal structure and analyze the motion of the components and bones during movement, and (2)to examine the accuracy of the measurement error at the methods of motion capture in this system.

\section{Methods}

\subsection{4-Dimensional Human Model for the Patient after THA}

We used skeletal structure data from CT (HiSpeed CT, GE Medical Systems, Milwaukee, WI). 15 reflective markers for infrared-light were attached to characteristic points on the body surface of the patient and CT images were obtained of the whole lower limb. Then 3-D model of each bone was reconstructed from that CT dataset. To achieve accurate model with minimum exposure, we scanned joint periphery part with $3 \mathrm{~mm}$ intervals and the other part with $5 \mathrm{~mm}-30 \mathrm{~mm}$ intervals. Accuracy of the model around the artificial joint falls off from the influence of the metal artifact, therefore we registered the CAD data of the patient's implant to the reconstructed 3-D model using the algorithm of 3-D surface registration (ICP algorithm[7]).

Next we acquired motion capture data of the patient. We conducted motion capture by tracking reflective markers attached to the skin using the VICON system, and regarding the motions that we could not capture due to occlusion, we used magnetic 3-D position sensors (FASTRAK, Polhemus, USA). By acquiring the relative positions between bones and markers from CT data, the movement of the skeletal structures could be calculated from the movement of the markers. As a result, we visualized the skeletal movement on the basis of this data, and analyzed various motion parameters such as hip joint angle, range of motion, impingement point between bones and/or implants and so on. We also calculated the distance that each point on the femoral head component moves over the sliding surface of the acetabular component during one motion cycle, because this distance may exert some influence on the extent of the wearing of the sliding surface.

In order to easily recognize the correlation between the skeletal movement and the patient's behavior, we superimposed the skeletal model onto the video footage that was captured by digital video camera in sync with the motion capture system. For the purpose of displaying the video footage and the skeletal model on the same coordinate system, estimating the camera parameter, including the position and orientation of the camera and the focus length or the principal point of the camera coordinate, is necessary. We obtained these information by using a camera calibration algorithm[8] from the correspondence of the skin marker position on the $3 \mathrm{D}$ coordinates with the $2 \mathrm{D}$ image coordinate. By superimposing the skeletal model onto the video footage, we were able to observe the movement of the skeletal structures and patient's outward appearance simultaneously. 


\subsection{Examination of the Accuracy}

We carried out an experiment to examine the accuracy of this system. The accuracy of the joint angle measurements in this system mainly depends on the rigidity between the reflective markers and the underlying bones, and therefore movements of the skin markers against the bones during motions are the source of measurement errors. To validate these measurement errors, relative positions of the skin markers against the bones in various postures was evaluated using an open MRI (SIGNA SP 0.5 T, GE Medical Systems, Milwaukee, WI).

5 reflective markers were attached to the volunteer's skin and MR images (SIGNA Horizon LX HiSpeed 1.5T, GE Medical Systems, Milwaukee, WI) were obtained from the pelvis to the femur to acquire the bone structure and the relative position of the reflective markers. As well, 3-D models of the bones and markers were reconstructed from MRI data. Next the volunteer with the reflective markers was ordered to rest for 12 static postures for a few minutes in the valid area of the open MRI, and the structure data of the bones around the hip was obtained. Simultaneously, the positions of the markers were also tracked and captured by the VICON sensor. By matching the 3-D models from the open MRI images to the models from the MRI images for each posture, the relative position between the pelvis and the femur was obtained and was used for the gold standard of measurements. The relative position between the pelvis and the femur was also calculated by combining the 3-D models with the data of marker position captured by the VICON sensor for each posture. After comparing these data to the gold standard data, we estimated the errors in the measurement by skin marker.

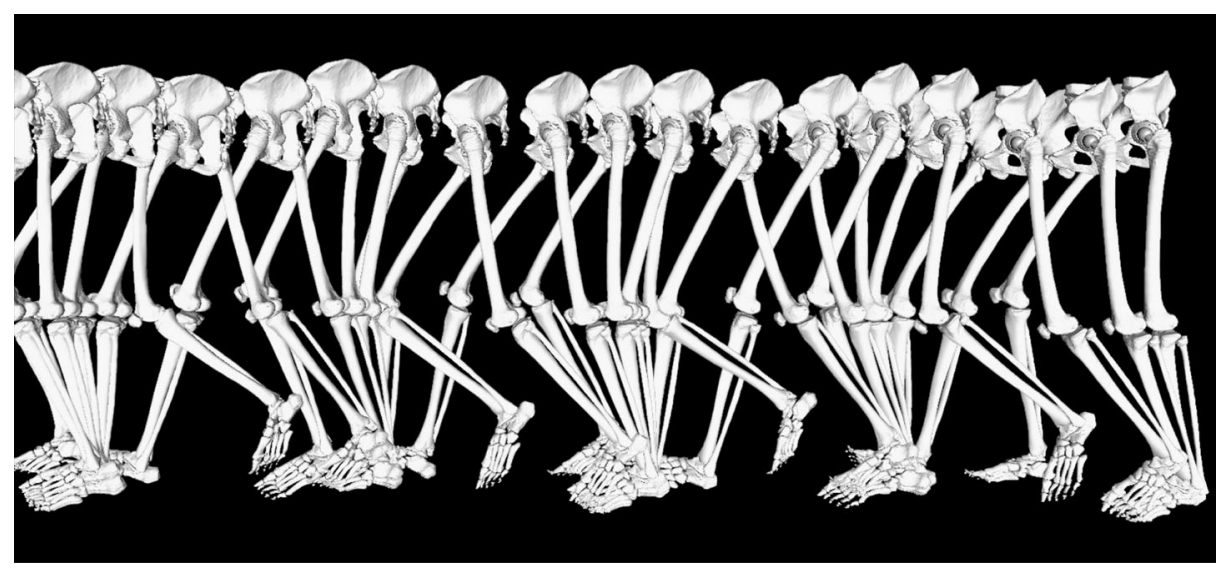

Fig. 1. Time sequential images of the patient's skeletal structures during walking

\section{Result}

Fig. 1 shows the time sequential images of the patient's (female, age 51yr, height $150 \mathrm{~cm}$, weight $44 \mathrm{~kg}$ ) skeletal structures of whole lower limb during walking. Fig.2, Fig.3 and Fig.4 show the motion analysis using this system while getting up and down from a chair. The height of the chair was adjusted to be equal to the distance from the 
head of fibula on the operated side to the floor. Fig.2 represents the display of this system. By displaying bones transparently, a clinician can grasp the movement of the components during motions intuitively. Fig. 3 represents the blended images of the patient's skeletal model and the captured video footage. Fig. 4 shows the estimated distribution of the extent of the wearing and the safe range of hip motion. Fig.5 indicates the measurement errors of the hip joint flexion angle. For each static posture, the measurement errors of the hip angle using current system were within about \pm 10 degrees for flexion, adduction, and internal rotation.

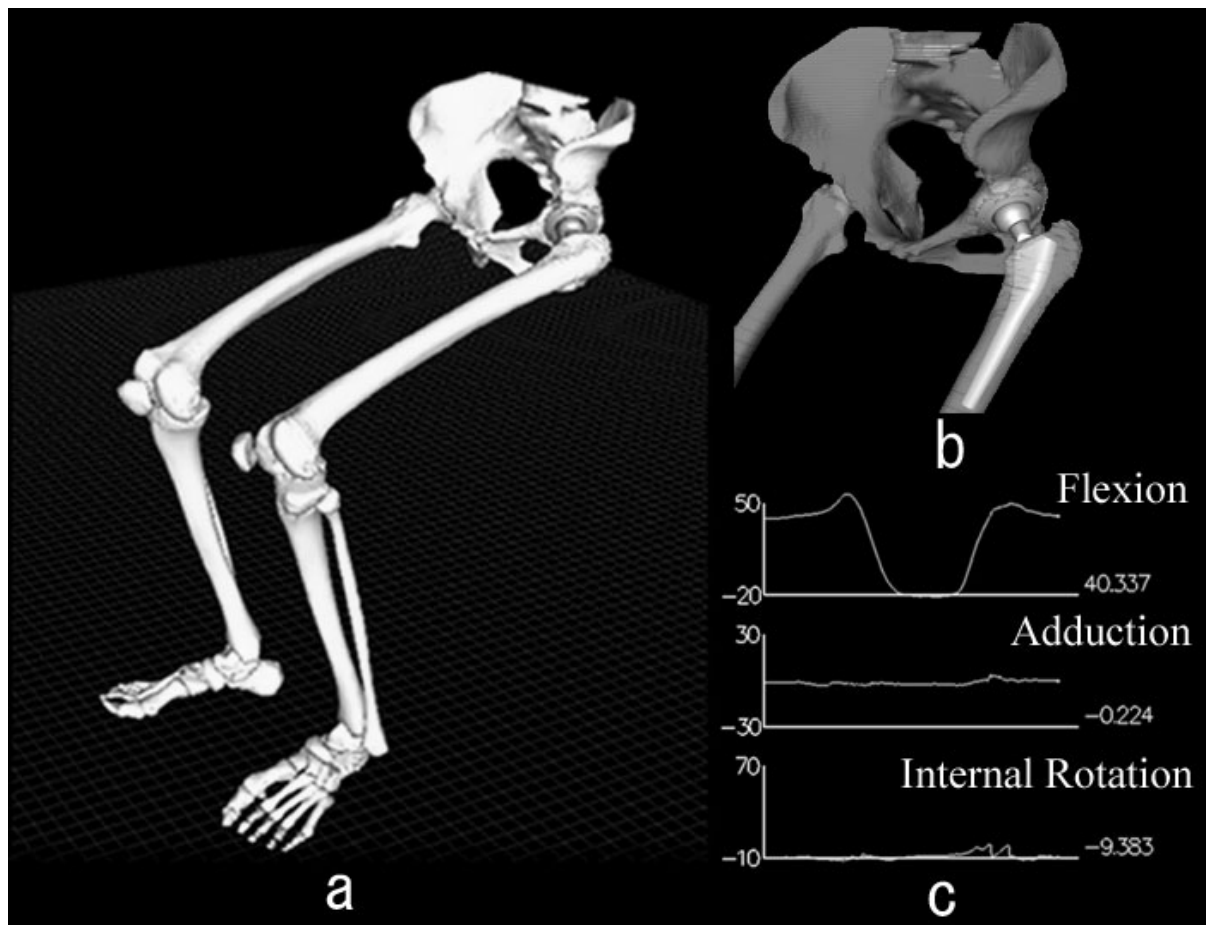

Fig. 2. The display of this system. (a) indicates the skeletal structures of the whole lower limb during sitting. (b) depicts geometry and orientation of the components. (c) shows the time sequential graph of the hip joint angle during sitting

\section{Conclusion}

Daily motion of the patients who underwent THA has been limited mainly based on intraoperative findings about hip stability in order to prevent complications of the hip joint in postoperative daily motion. However, it is difficult to give precise guidance for each patient in postoperative daily motion in terms of individual differences of alignment of components, skeletal structure and characteristics of daily motion.

This system revealed the movement of the patient's skeletal structures and components during various motions by integrating the patient specific 3-D model from CT 
data and the patient's motion capture data. From this model we could recognize the movement of the components intuitively and also quantitatively. Consequently, this system enables dynamic analysis in terms of not only alignment of components and bones of each patient but also individual differences of the characteristics of daily motion.

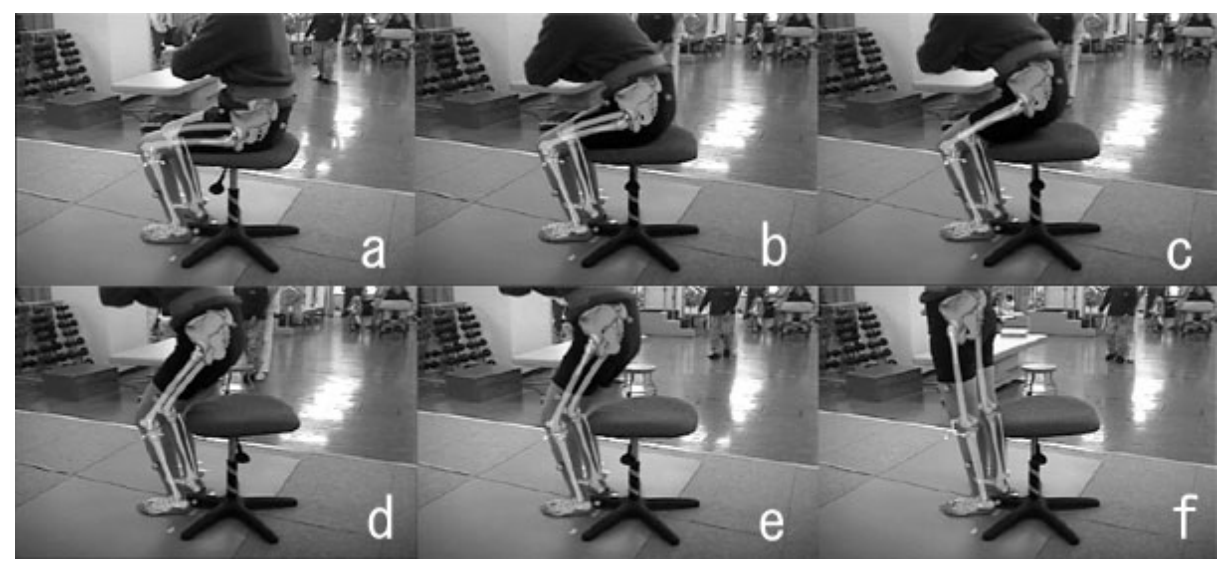

Fig. 3. Blended images of the patient's skeletal model and the captured video footage while getting up and down from a chair. The images laid out time sequentially (a) to (f). These images are visualizing the inner skeletal structures of the patient and his outward appearance simultaneously

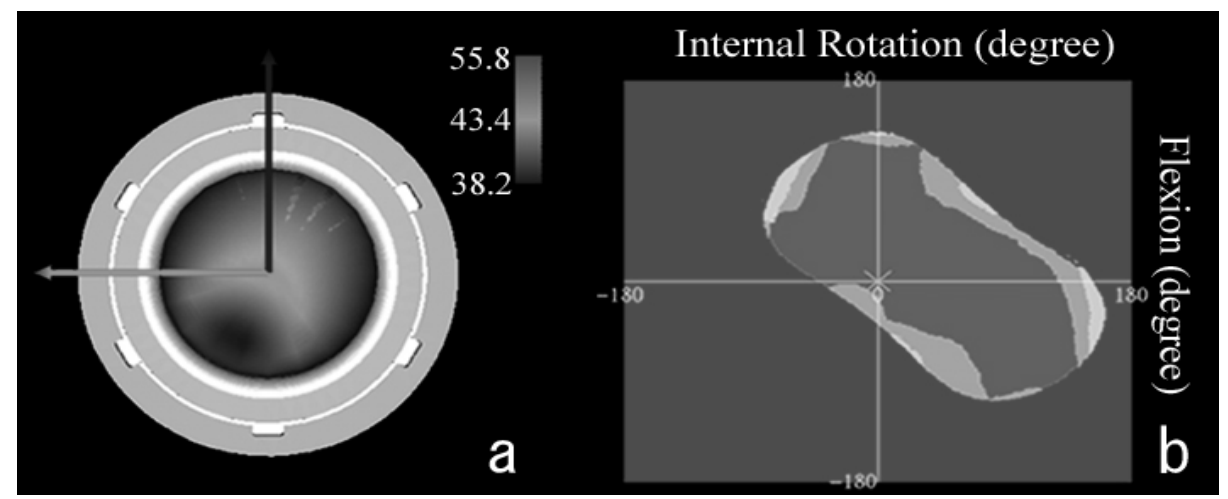

Fig. 4. (a) shows estimated distribution of the extent of the wearing at the sliding surface of the acetabular component while the patient get up and down from a chair. (b) indicates the safe range of hip motion at the movement on the sagittal plane

By using several applications, by which we can calculate various motion parameters of the hip joint, we were able to assess the safe range of component alignment for the hip dislocation and predict the wearing of the sliding surface on the acetabular implant. 


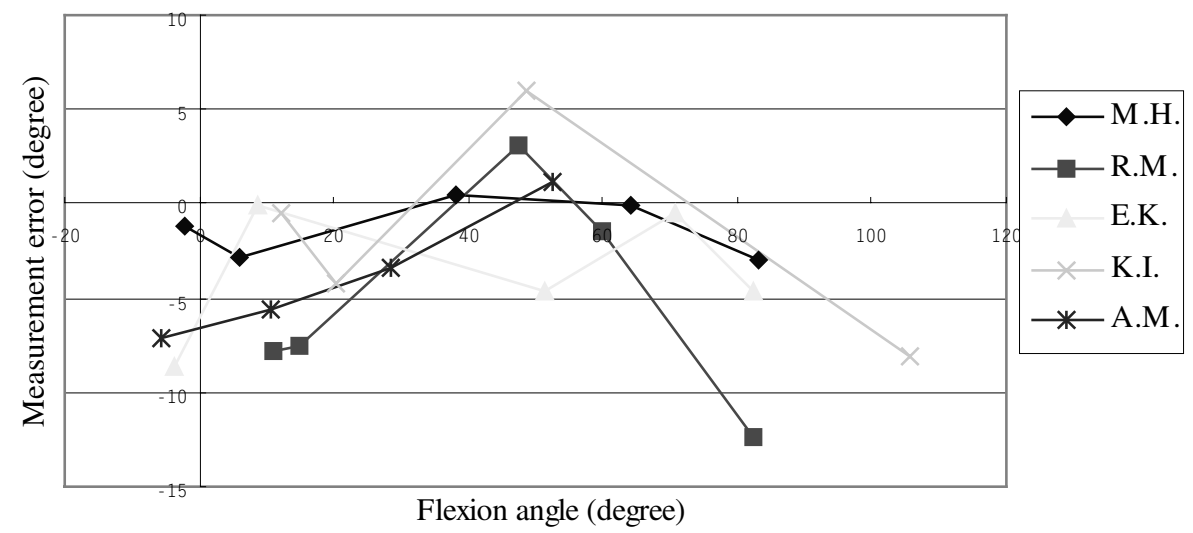

Fig. 5. Measurement errors of the flexion angle. The flexion angles from -5 degrees to 105 degrees are examined, and the errors are within about \pm 10 degrees. Almost the same tendency was acquired for adduction and internal rotation angle

The maximum degree of the hip angle measurement error for the current system was within $\pm 10^{\circ}$ for flexion, adduction, and internal rotation. From the viewpoint of the degree of angle, reducing the error wherever possible is preferable. However, as long as the skin marker is used, the error induced by the changes of the marker position with respect to the underlying bones is inevitable. On the other hand, currently the method by using skin markers is the only way to capture human motion noninvasively. And according to a clinician, this degree of error is quite acceptable to enable the use of this system in a clinical setting to provide useful information regarding each patient and the corresponding safe daily motion to prevent complications of the hip.

This system helps the patient to understand the state of the components in his body, and also helps clinicians to give advice regarding the limitations of daily motion to the patient. More investigation with this system will enable to estimate the safe motion preventing hip dislocation precisely, and lead to more precise guidance of each patient in postoperative daily motion.

\section{References}

1. Richard C. Johnston, Gary L. Smidt: Hip Motion Measurements for Selected Activities of Daily Living, Clinical Orthopaedics and Related Research Number 72 (1970) 205-215

2. Ronald Y. G. Woo, Bernard F. Morrey, Rochester, Minnesota: Dislocations after Total Hip Arthroplasty, The Journal of Bone and Joint Surgery vol. 64-A No.9 (1982) 1295-1306

3. F. Pierchon, G.Pasquer, A. Cotton, C. Fontaine, J. Clarisse, A. Duquennoy: Causes of Dislocation of Total Hip Arthroplasty, The Journal of Bone and Joint Surgery vol. 76-B No.1 (1994) 45-48

4. A.Wang :A unified theory of wear for ultra-high molecular weight polyethylene in multidirectional sliding, Wear 248 (2001) 38-47 
5. D'Lima DD.,et al.: The effect of the orientation of the acetabular and femoral components on the range of motion of the hip at different head-neck ratios, The Journal of Bone and Joint Surgery 82- A (2000) 315-321

6. Anthony M. DiGioia, et al: Image Guided Navigation System to Measure Intraoperatively Acetabular Implant Alignment. Clinical Orthopaedics and Related Research 355 (1998) 822

7. P.J. Besl, and N.D. McKey: A method for registration of 3-D shapes, IEEE Trans. Patt. Anal. Machine Intell. vol. 14 no.2 (1992) 239-256

8. Roger Y. Tsai: A Versatile Camera Calibration Technique for High-Accuracy 3D Machine Vision Metrology Using Off-the-Shelf TV Cameras and Lenses, IEEE Journal of Robotics and Automation vol. RA-3 No.4 (1987) 323-344

9. Y. Otake, Eun S. S., N. Suzuki, A. Hattori, Y. Yamamoto, M. Abo, S. Miyano: Development of 4-Dimensional Whole Body Musculoskeletal Model "Digital Dummy", The 1st World Congress of the International Society of Physical and Rehabilitation Medicine (2001) 47-52 